Users may view, print, copy, and download text and data-mine the content in such documents, for the purposes of academic research, subject always to the full Conditions of use: http://www.nature.com/authors/editorial_policies/license.html\#terms

Correspondence: Michael L. Nickerson, Ph.D., Laboratory of Translational Genomics, National Cancer Institute, 8717 Grovemont Circle, Rm. 225C, Bethesda, MD 20892-4605. Phone: 301-451-2724; Fax: 301-402-3134; nickersonml@ mail.nih.gov.

${ }^{14}$ Current address: Laboratory of Translational Genomics, National Cancer Institute, Bethesda, MD, USA

SUPPLEMENTARY INFORMATION

Supplementary Information accompanies the paper on the Oncogene website (http://www.nature.com/onc).

Conflict of Interest: The authors declare no conflict of interest. The contents of this publication does not necessarily reflect the views or policies of the Department of Health and Human Services, nor does mention of trade names, commercial products, or organizations imply endorsement by the U.S. government. The funders had no role in study design, data collection and analysis, decision to publish or preparation of the manuscript. 
${ }^{12}$ Protein Expression Laboratory, Leidos Biomedical Research, Inc., Frederick National Laboratory for Cancer Research, Frederick, MD, USA

${ }^{13}$ School of Medicine, Johns Hopkins University, Baltimore, MD, USA

\section{Abstract}

Genetic alterations associated with prostate cancer $(\mathrm{PCa})$ may be identified by sequencing metastatic tumor genomes to identify molecular markers at this lethal stage of disease. Previously, we characterized somatic alterations in metastatic tumors in the methylcytosine dioxygenase teneleven translocation 2(TET2), which is altered in 5-15\% of myeloid, kidney, colon and prostate cancers. Genome-wide association studies previously identified non-coding risk variants associated with $\mathrm{PCa}$ and melanoma. We performed fine-mapping of PCa risk across TET2 using genotypes from the PEGASUS case-control cohort and identified six new risk variants in introns 1 and 2. Oligonucleotides containing two risk variants were bound by the transcription factor octamer-binding protein 1 (Oct1/POU2F1) and TET2 and Oct1 expression were positively correlated in prostate tumors. TET2 is expressed in normal prostate tissue and reduced in a subset of tumors from the Cancer Genome Atlas (TCGA). Small interfering RNA (siRNA)-mediated TET2 knockdown (KD) increases LNCaP cell proliferation, migration, and wound healing, verifying loss drives a cancer phenotype. Endogenous TET2 bound the androgen receptor (AR) and AR-coactivator proteins in LNCaP cell extracts, and TET2 KD increases prostate-specific antigen (KLK3/PSA) expression. Published data reveal TET2 binding sites and hydroxymethylcytosine (hmC) proximal to $K L K 3$. A gene co-expression network identified using TCGA prostate tumor RNA-sequencing identifies co-regulated cancer genes associated with 2oxoglutarate (2-OG) and succinate metabolism, including TET2, lysine demethylase (KDM) $K D M 6 A$, BRCA1-associated $B A P 1$, and citric acid cycle enzymes IDH1/2, SDHAß, and $F H$. The co-expression signature is conserved across 31 TCGA cancers suggesting a putative role for TET2 as an energy sensor (of 2-OG) that modifies aspects of androgen-AR signaling. Decreased TET2 mRNA expression in TCGA PCa tumors is strongly associated with reduced patient survival indicating reduced expression in tumors maybe an informative biomarker of disease progression and perhaps metastatic disease.

\section{Keywords}

cancer risk variant; androgen signaling; metabolic sensor; epigenetics; oxoglutarate

\section{INTRODUCTION}

Metastatic prostate cancer ( $\mathrm{mPCa}$ ) is poorly controlled using existing therapies and is responsible for more than 258,000 deaths worldwide each year. ${ }^{1}$ Molecular markers that distinguish indolent from aggressive disease and that identify therapeutic targets may improve patient stratification for precision medicine. Genetic analysis to determine the precise molecular chronology of causal alterations arising during progression of prostate cancer (PCa) to castration-resistant and metastatic disease has been difficult, in part, because of the very high heterogeneity of primary adenocarcinomas and a paucity of metastatic tumors. 
Frequent somatic ten-eleven translocation 2(TET2) alterations have been observed in myeloproliferative disorders (MPDs), ${ }^{2,3}$ mastocytosis and polycythemia vera, and in fewer but significant numbers of epithelial-derived tumors, ${ }^{4-11}$ including PCa. ${ }^{9-11}$ Germline TET2 variants are associated with an increased risk of prostate ${ }^{12,13}$ and endometrial ${ }^{7}$ cancers, and melanoma. ${ }^{8}$ Thus, germline and somatic alterations implicate $T E T 2$ as a cancer gene in MPDs and epithelial cancers.

Previously, we sequenced the exomes of five distinct metastatic tumors and healthy tissue from a patient with $\mathrm{PCa}$ and identified four cancer gene alterations, ${ }^{11}$ an inherited breast cancer-1 (BRCA1) truncation (p.E23fs*) associated with $\mathrm{PCa},{ }^{14}$ a somatic deletion giving rise to a fusion of the transmembrane protease TMPRSS 2 and the transcription factor (TF) $E R G,{ }^{15}$ a somatic missense substitution in a bromodomain of $P B R M 1,{ }^{16}$ and a somatic TET2 missense substitution (p.P562A). Significantly, the TET2 substitution was observed in all $11 \mathrm{mPCa}$ tumors but not in the primary tumor, suggesting TET2 may provide a survival benefit distinct from altered BRCA1, TMPRSS2-ERG, and PBRM1. ${ }^{17}$ Analysis of mPCa tumors from additional patients showed TET2 alterations in 13/30 tumors ${ }^{11,18}$ and we identified a frameshift truncation (p.T229fs*) in DU145, an androgen-independent cell line derived from an $\mathrm{mPCa}$ brain tumor. Thus, the presence of germline and somatic TET2 alterations suggests an altered TET2 may be associated with progression in a subset of $\mathrm{PCa}$ patients.

In this study, we identify six new PCa risk variants in TET2 introns 1 and 2, and show TET2 physically interacts with the androgen receptor (AR) and AR-coactivators PSPC1, NONO and SFPQ. TET2 loss drives a cancer phenotype by increasing LNCaP prostate cell proliferation and invasion, and KLK3/PSA expression. Network analysis reveals TET2-AR interacts with proteins that are frequently altered across cancers. We identify a TET2associated co-expression signature in TCGA PCa tumors that includes cancer genes encoding functions related to 2-OG and succinate metabolism. The signature is observed across cancers indicating frequent dis-regulation of 2-OG and succinate metabolism in cancer.

\section{RESULTS}

\section{Prostate cancer risk variants}

We genotyped 47 TET2 locus single nucleotide polymorphisms (SNPs) in 4,838 cases and 3,053 controls in the PEGASUS cohort as part of the Cancer Genetic Markers of Susceptibility Study (National Cancer Institute, Bethesda, MD) (Supplementary Table S1). Seven, including the previously reported promoter variant, rs7679673 ${ }^{12}$ and six new SNPs in introns 1 and 2, were found to be significantly associated with increased PCa risk ( $\left.\mathrm{p} \leq 10^{-4}\right)$ (Table 1, Figure 1a). SNP rs7679673 retained the highest association with risk ( $\mathrm{p}=1.6 \mathrm{x}$ $\left.10^{-6}\right)$ followed by rs 1015521 in intron $2\left(\mathrm{p}=8.6 \times 10^{-5}\right)$. Two SNPs in intron 1, rs 17508261 and rs6825684, had a slightly more protective homozygous odds ratio ( 0.68 and 0.69 , respectively) than rs7679673 (0.72).

We examined TF binding to oligonucleotides containing the new risk SNPs by electrophoretic mobility shift assay and observed protein binding to oligonucleotide probes 
containing rs 17508261-C and rs7655890-G/T in LNCaP and PC3 cell line nuclear extracts (Figure 1b). TF binding was confirmed in repeat experiments and additional proteins interacting with these oligonucleotides in kidney 293, cervical HeLa, and breast MCF7 cells were observed but not further examined (Figure 1c). In silico analysis of TF-binding sites revealed that rs17508261 was located in an Oct1/POU2F1 ${ }^{19}$-binding DNA sequence motif (Supplementary Figure S1). Supershift analysis with TF-specific antibodies showed altered migration or reduced binding to labeled oligonucleotides containing rs 17508261-C and rs7655890-T in the presence of an anti-Oct1 antibody whereas no supershift was observed with other TF antibodies examined. The SNP genotypes indicate rs17508261-C and rs7655890-T are risk and protective alleles, respectively (Figure 1e; Supplementary Tables S1, S2). Thus, rs17508261-C may be a functionally significant PCa risk variant due to Oct1 binding.

Three risk variants, rs7679673-A, rs17508261-C, and rs7655890-G, were in linkage disequilibrium in a rare risk haplotype (Supplementary Table S2). To test the effect of these alleles on TET2 expression, we genotyped rs7679673, rs17508261, and rs7655890 in eleven PCa cell lines and examined normalized TET2 expression. We found no association between the SNP genotype and TET2a mRNA expression (Supplementary Figure S2), but did observe that 4 of 11 (36\%) PCa cell lines, DU145, PC3, PWR-1E, and VCaP, exhibited significantly reduced TET2 expression (Figure 1f; Supplementary Tables S3, S4). TET2 expression in DU145 cells may be reduced due to a p.T229fs* mutation. ${ }^{11}$

To analyze the relationship between TET2 and Oct 1 expression, we measured the mRNA levels of TET2 and Oct1 in 12 prostate cancer cell lines using real-time, quantitative PCR (QPCR) (Supplementary Figure S3). Pearson correlation showed a positive but not significant correlation between TET2 and Oct1 expression. We examined TET2 and Oct1 expression in an independent dataset, TCGA PCa tumors, using tumor RNA sequencing and observed a positive Pearson correlation indicating co-expression ( $\mathrm{p}=0.0097$, Supplementary Figure S3). Thus, risk SNPs within the TET2 transcriptional locus conclusively identify $T E T 2$ as the PCa risk gene on chr 4q24. The significance of Oct1-binding to TET2 risk variants relative to Oct1's described roles in cancer stem cells and the cell cycle remains to be determined. ${ }^{19,20}$

\section{Somatic alterations in epithelial cancers}

The spectrum of somatic mutations indicates that TET2 functions as a tumor suppressor in MPD, ${ }^{2,3}$ so we assembled 97 somatic mutations observed in epithelial tumors from published studies, ${ }^{4-11}$ the Cancer Genome Atlas (TCGA), and the Catalogue of Somatic Mutations in Cancer (COSMIC) (Supplementary Figure S4a; Supplementary Table S5). Forty-nine (51\%) alterations were predicted to be loss of function, indicating that TET2 potentially functions as a tumor suppressor in epithelial cancer. Additional experimental data is required to confirm whether all alterations have a similar effect on function. Recent next generation sequencing (NGS)-molecular analyses of $\mathrm{PCa}^{9-11,21,22}$ available through the cBioPortal for Cancer Genomics ${ }^{23}$ reveal a low but significant number of somatic TET2 alterations (Supplementary Figure S4b). We were able to assess TET2 status in 246 primary adenocarcinomas and 117 metastatic tumors and observed a significantly greater number of 
somatic alterations in metastatic as compared to primary tumors (23 [20\%] and 14 [6\%], respectively; Fisher's exact test $\mathrm{p}=4.7 \times 10^{-4}$ ). Additionally, several studies identified tumors with focal or homozygous loss of TET2 (Supplementary Figure S4c). 5,11,24

\section{Reduced expression in advanced, lethal prostate cancer}

Reduced TET2 expression in MPD is a predictor of disease progression and poor overall survival. ${ }^{25,26}$ We re-examined normalized mRNA expression in a recent PCa study ${ }^{9}$ of 29 matched normal adjacent tissue (NAT) samples, 131 primary tumors, and 19 metastatic tumors. Expression decreased when comparing the NAT with primary tumors but not significantly, but was significant when comparing primary with metastatic tumors $(\mathrm{p}=0.01$; Figure 2a). Expression was reduced in 145 primary and metastatic tumors with a high $(\geq 7)$ versus low $(<7)$ combined Gleason score $(\mathrm{p}=0.006$; Figure $2 \mathrm{~b})$. There was no association between reduced TET2 expression and tumor stage ( $\mathrm{p}=0.44$, data not shown).

We evaluated the normalized TET2 mRNA expression in tumors compared to the NAT using a Z-score $\leq-2.0$ criteria, and identified 7 of 131 (5.3\%) primary tumors and 7 of $19(36.8 \%)$ metastatic tumors with significantly reduced expression ('Low') as compared to the average (Figure 2c). Reduced expression in 3 of 14 (21\%) TET2-low tumors were attributable to copy number variation (CNV) loss; gene sequencing data were not available. ${ }^{9}$ Thus, unknown additional mechanism(s) reduce mRNA expression in the majority of tumors. Retrospective Kaplan-Meier analysis revealed shortened disease-free survival (DFS) in the seven patients with TET2-low primary tumors as compared with 123 patients with tumors displaying average TET2 expression ( $\mathrm{p}=6.4 \times 10^{-6}$; Figure $2 \mathrm{~d}$ ). Thus, reduced TET2 mRNA expression in tumors is significantly associated with a lethal subtype of PCa.

We confirmed by real time-polymerase chain reaction and Western blot (WB) using a previously described monoclonal antibody ${ }^{27}$ that TET2a (NM_001127208; 2002 amino acids [aa]) was highly expressed in prostate tissue, tumors, and cell lines. TET2a and TET2b (NM_017628; 1165 aa) were both expressed in prostate samples and are transcribed 810 base pair (bp) apart (HG19) (Figure 1a, 2e-h; Supplementary Table S4). All samples exhibited alternative splicing of the TET2a untranslated exon 2 (TET2a-deleted exon 2 [TET2a-delex2]).

Immunohistochemistry (IHC) of serial sections of a PCa tissue microarray revealed TET2 and 5-hydroxymethylcytosine $(\mathrm{hmC})$ were depleted in tumors as compared to the NAT (Supplementary Figure S5). We observed TET2- and hmC-positive epithelial cells and TET2-negative, hmC-positive stromal cells. The stromal cells exhibiting 5-hmC staining suggests TET1 or TET3 may be active. TET2 was observed in the cytoplasm, indicating likely cytoplasmic-to-nuclear shuttling similar to that previously observed for several TET2AR nexus proteins described below, including the AR, BAP1, and the E1A binding protein p300 (EP300).

\section{TET2 loss is associated with cancer progression}

We identified two siRNAs (Figure 1a; Supplementary Table S3) that reduced TET2 protein levels by $>60 \%$ in both LNCaP and DU145 cells, comparable to the reduction observed in tumors. Treatment of LNCaP cells with siRNA siTET2-1 reduced TET2 protein levels by 
$63 \%$ ( $37 \pm 6$ and $100 \pm 27$, respectively) after 24 hours as compared with untreated cells (Figure 3a-c) [normalized mRNA expression was reduced by $41 \%$ as compared with untreated $\mathrm{LNCaP}(18 \pm 1$ and $30 \pm 1$, respectively)]. Treatment with a non-targeting scrambled-siRNA (scRNA) did not significantly alter TET2 expression.

In vitro, TET2 KD after siTET2-1 treatment increased LNCaP cell proliferation by $200 \%$ after $24 \mathrm{~h}$ as compared with untreated cells $\left(1 \times 10^{6}\right.$ cells vs. $5 \times 10^{5}$ cells, respectively) (Figure 3d). Scrambled siRNA treatment did not alter proliferation $(\mathrm{p}=0.36)$. Similarly, TET2 KD increased the proliferation of androgen-independent DU145 cells by $193 \%$ (Supplementary Figure S6). TET2 KD in LNCaP cells revealed rapid wound healing as compared with untreated cells after $48 \mathrm{~h}(49.5 \%$ and $23.5 \%$ closure, respectively) (Figure $3 \mathrm{e}$ and f). After TET2 KD, we observed LNCaP cells migrating into the wound area, and this migration was independently confirmed by multiple experiments. TET2 KD increased LNCaP cell transwell invasion through matrigel in Boyden chambers by $236 \%$ as compared to untreated cells ( $123 \pm 30$ and $52 \pm 19$ cells, respectively) (Figures $3 \mathrm{~g}$ and h). Colony formation in soft agar did not differ between comparison groups $(\mathrm{p}=0.22$; Supplementary Figure S6). Thus, reduced TET2 mRNA and protein increase in vitro prostate cell proliferation, wound healing, and invasion; characteristics favoring cancer progression and metastasis.

\section{TET2 binding proteins in HEK293T cells}

We purified TET2 binding proteins from human embryonic kidney 293T (HEK293T) cells using exogenous $3 x$ FLAG-tagged TET2 (FLAG-TET2) and affinity purification and mass spectrometry (MS) (Supplementary Tables S6 and S7). We identified the acetylglucosamine transferase OGT, which catalyzes the transfer of acetylglucosamine to histones. ${ }^{28,29}$ Putative TET2 interactors were enriched in proteins with RNA and chromatin binding functions. Several AR binding proteins were detected, including PSPC $1,{ }^{30}$ filamin $\mathrm{A}^{31}$ and KDM6A. ${ }^{10,32,33}$ Additionally, peptides from AR-coactivators, the octamer-binding NONO, and the splicing factor SFPQ were enriched in TET2 affinity purifications as compared to the controls (Supplementary Figure S7). PSPC1, NONO and SFPQ bind the AR to regulate transcription; ${ }^{34}$ however, AR peptides were not observed. Using forward and reverse immunoprecipitation (IP), we confirmed TET2 interactions with PSPC1, OGT, and NONO in HEK293T cells.

\section{TET2-AR binding in prostate cells}

We confirmed the interactions between endogenous TET2 and OGT, PSPC1, NONO and SFPQ in LNCaP cells using IP with the appropriate antibodies (Abs) (Figure 4a). Reciprocal IP of endogenous TET2 with anti-TET2 Ab confirmed the interactions (Figure 4b). Anti-AR $\mathrm{Ab}$ precipitated TET2, NONO and OGT in LNCaP cells (Figure 4c). Additional IPs in HEK293T cells failed to confirm a TET2-AR interaction (data not shown), suggesting it may be specific to prostate cells.

SFPQ and NONO regulate AR-mediated gene expression by recruiting the transcription regulator SIN3A. SIN3A was detected by WB after IP with anti-AR Abs (Figure 4c); however, we did not detect a TET2-SIN3A interaction (data not shown). Treatment of cells 
with DHT followed by IP with anti-AR Abs, and WB with anti-TET2 or anti-SFPQ Abs showed that interactions in the presence of DHT are slightly (SFPQ) or completely reduced (TET2) when DHT is removed (Figure 4d). Experiments were independently replicated and not due to a DHT effect on protein in the lysates (data not shown). Thus, a DHT-sensitive, SIN3A-independent, TET2-AR complex is observed in LNCaP cells.

\section{TET2 loss alters expression of genes associated with prostate cancer}

A TET2-AR interaction suggests a potential role in regulating the expression of genes associated with $\mathrm{PCa}$, possibly through $\mathrm{hmC}$ formation. ${ }^{25-27}$ We examined the expression of 53 genes in LNCaP cells after TET2 KD by siTET2-1 using QPCR to determine whether the expression of genes known to be responsive to androgen ${ }^{35}$ or genes encoding AR pathway proteins (AR signaling array, Bio-Rad, Hercules, CA, USA) are altered, either of which could alter androgen signaling. The expression of 24 genes were excluded due to significant differences in expression between untreated and scRNA-treated cells (two-tailed T-test $\mathrm{p}<0.05$ ), leaving 29 assays in the final analysis. Of these, 12 genes had a significant expression change (p-value $<0.05$ and/or |fold change| of at least 1.1) (Supplementary Table S8). Comparing gene expression between scRNA and siTET2-1 treated cells, TET2 expression was the most significantly reduced, by 1.7 fold $\left(\mathrm{p}=1.9 \times 10^{-7}\right)$. The expression of six genes was increased after TET2 KD, including KLK3PSA, ${ }^{36}$ hydroxyprostaglandin dehydrogenase $H P G D$ and a centromeric nucleosome-associated protein $C E N P N$. The expression of five additional genes was decreased, including an extracellular signalregulated kinase $M A P 2 K 1$, the glycogen synthase kinase $G S K 3 B$ and the GSK3B-complex protein beta-catenin $(C T N N B 1)$.

We confirmed the effect of TET2 KD using a second independent siRNA (siTET2-4) recently described by Takayama et al. ${ }^{37}$ (Figure 1a; Supplementary Table S3). We performed three independent treatments of LNCaP cells with siTET2-4 or a GC-matched scrambled siRNA control (scRNA) in the absence and presence of DHT. Analysis of quantitative Western blots reveal a 52\% reduction of TET2 protein compared to scRNA treated cells, similar to the KD achieved using siTET2-1 (Figure 5a). Unfortunately, a key transfection reagent (siGene, Promega, Japan) was not available to obtain a more effective KD achieved by Takayama et al. ${ }^{37}$ Comparing scRNA-treated cells without DHT to scRNA-treated cells with DHT showed DHT treatment decreased TET2 protein by 33\%, confirming previous observations. ${ }^{37}$ PSA protein expression was significantly increased ( $\mathrm{p}<0.05$; Figure $5 \mathrm{~b}$ ), confirming the gene expression results (Supplementary Table S8) are correlated with increased protein using two distinct siRNAs targeting TET2. DHT treatment further increased PSA as expected independent of TET2 status.

The expression changes after TET2 KD indicate TET2 may regulate the expression of a subset of androgen-responsive genes. ${ }^{35}$ We investigated whether there were TET2 binding sites and hmC proximal to genes with expression changes in Supplementary Table S8 using recently published chromatin immunoprecipitation-sequencing data from LNCaP cells. ${ }^{37}$ TET2 binding sites were identified near each gene examined that were accompanied in a more regional genomic context by hmC and methylcytosine (mC) in LNCaP cells. AR binding sites were observed near these genes in sequencing data from DHT-treated prostate 
cell line, BicR (Supplementary Figure S8, Supplementary Table S9). Thus, TET2 KD increases the expression of PSA, an androgen-AR responsive biomarker, in the same direction as DHT treatment, indicating TET2 has a role in regulating the expression of at least a subset of androgen-AR responsive genes (Figure 5c).

\section{TET2-AR interact with cancer proteins}

TET2 and AR are encoded by frequently altered cancer genes (Supplementary Figure S9) and published protein-protein interactions reveal a 'nexus' of interacting cancer gene products (cancer proteins) with diverse functions: epigenetic (left), basal transcription (middle), and 'output' to mRNA splicing and the cell cycle (right) (Figure 6, Table 2, Supplementary Table S10). The nexus includes the oncogenic proteins AR and cyclin D1, and multiple tumor suppressors associated with DNA and RNA transcription (Figure 6b). Cancer proteins in multiple signaling pathways converge on the nexus, including androgen signaling (AR, ${ }^{10,38,39} \mathrm{KDM6A},{ }^{10,32,33}$ TET2), DNA repair (BAP1 ${ }^{10,40,41}$ ), insulin signaling (OGT) and oxygen sensing (Figure 6c). The distribution of somatic alterations in a recent PCa study ${ }^{10}$ indicates the nexus is targeted by largely mutually-distinct alterations across nexus genes in a majority (72\%) of tumors, most frequently by altered $A R$ and nuclear receptor coactivator 2 (NCOA2) (Supplementary Figure S9e). Core nexus proteins physically interact with additional cancer proteins as identified by the COSMIC Cancer Gene Census (Figure 6d).

We examined tumor gene co-expression networks based on RNA-sequencing from tumors for TCGA PCa tumors and 30 additional cancers to identify transcriptional modules correlated with TET2. Similar analyses have identified the transcriptional responses of cells to changing conditions and identified co-regulated interacting proteins to provide mechanistic insight underlying complex cellular processes. ${ }^{42,43}$

The analysis confirmed the inversely-correlated expression in PCa tumors between TET2 and $K L K 3$ (as observed above), CTNNB1, GSK3B, and the cyclic-AMP dependent kinase $P R K A C B$, as we observed in $\mathrm{LNCaP}$ cells after TET2 KD (Figure 6e, Supplementary Figure S10a). We did not observe co-expression between TET2 and the majority of androgen-AR genes by the analysis similar to our experimental results after TET2 KD in LNCaP cells, suggesting a likely distinct rather than general function for TET2 in androgen-AR signaling.

The primary role of TET2 is to catalyze $\mathrm{hmC}$ formation from $\mathrm{mC}$ in DNA. TET2 binds 2$\mathrm{OG}, \mathrm{O}_{2}, \mathrm{Fe} 2+$, and zinc as cofactors and produces succinate, potentially indicating a role for TET2 as an oxygen and redox-sensitive energy (metabolite) sensor. ${ }^{44,45}$ We examined the co-expression of genes encoding TET2-AR nexus proteins and proteins associated with 2OG and succinate metabolism (citric acid cycle enzymes ${ }^{46}$ ) and observed a robust signal (Figure 6f, Supplementary Figure S10b). The expression of genes encoding enzymes producing 2-OG (IDH1 and IDH $2{ }^{47}$ ) are inversely-correlated with the expression of genes encoding 2-OG consuming enzymes (TET1-3 and KDM6A). This transcriptional module is likely designed to tightly regulate and maintain 2-OG/succinate levels.

Genes co-expressed with TET2 encode additional TET-family enzymes TET1 and TET3, a large number of KDMs, and the oxygen $\left(\mathrm{O}_{2}\right)$ sensing components von Hippel-Lindau 
(VHL), ${ }^{46} \mathrm{HIF} 1$, ARNT and EGLN1 (Supplementary Figure S11). The $\mathrm{O}_{2}$ sensing components of the co-expression network likely reflect the regulation of oxygen levels by TET-enzymes $\left(\mathrm{O}_{2}\right.$ consuming dioxygenases) and hypoxia-responsive proteins such as HIF1 and ARNT. Significantly, the co-expression network comprised of TET2-AR nexus components and genes encoding 2-OG/succinate metabolizing enzymes that is observed in $\mathrm{PCa}$ is observed in all 31 TCGA cancers (Supplementary Figure S10c) indicating a conserved and frequently dis-regulated network across cancers.

\section{DISCUSSION}

This study implicates TET2 as a tumor suppressor in PCa that is altered by multiple mechanisms: germline noncoding risk SNPs and rare missense substitutions; ${ }^{11}$ somatic sequence changes and CNV (primarily loss); and reduced mRNA expression in tumors. The genetic complexity of TET2 alterations in PCa requires that multiple approaches be applied to understand causality to disease, which we have done using fine mapping of PCa risk, identification of binding proteins (AR), experimental TET2 KD combined with cell based and expression assays (PSA), and computational mining of protein interactions and tumor gene expression datasets.

Fine mapping revealed six SNPs in introns 1 and 2 that are significantly associated with PCa in the PEGASUS cohort, supporting TET2 as the causal gene associated with the promoter risk SNP rs $7679673 .{ }^{12}$ Somatic alterations in $6 \%$ of primary and $20 \%$ of metastatic tumors, reduced mRNA expression in a subset of tumors and experimental evidence that TET2 KD increases $\mathrm{LNCaP}$ cell proliferation and invasion indicate TET2 is a tumor suppressor in PCa. TET2 may have been overlooked as a PCa gene until this study because diverse mechanisms alter TET2 during disease progression and somatic CNV appears to be more frequent in less-studied metastatic PCa disease.

TET2 catalyzes hmC formation ${ }^{45}$ and future studies will examine global gene expression and the role of $\mathrm{hmC}$ in androgen-AR signaling. DNA $\mathrm{hmC}$ is catalyzed at developmentally associated enhancers ${ }^{48}$ that may mediate gene expression by a recently described genomic looping mechanism ${ }^{49}$ that may synthesize $\mathrm{hmC}$-modified RNA. ${ }^{50}$ TET2 alteration or loss in cancer may disrupt these genomic arrangements to dysregulate gene expression.

A role for TET2 in androgen-AR signaling is supported by the identification TET2 interaction with the AR and AR-transcriptional co-activators PSPC1, NONO and SFPQ by affinity chromatography-mass spectrometry and IP. Increased $K L K 3 / P S A$ mRNA and protein expression in LNCaP cells after treatment with two different siRNAs targeting TET2, and anti-correlated expression in TCGA PCa tumors by RNA NGS, confirms a role for TET2 in regulating PSA expression. Down-regulation of TET2 by DHT is in agreement with recent observations ${ }^{37}$ and indicates complex feedback mechanisms. The combination of an enzyme activity dependent on 2-OG and co-expression with 2-OG associated genes indicates TET2 is an energy sensor in a position to integrate 2-OG and androgen signals. We propose a quite reasonable hypothesize that TET2 modifies androgen-AR signaling based on the metabolic state (2-OG) of the cell. Experiments are planned to examine the underlying mechanism(s). 
A 2-OG co-expression module highly enriched in cancer proteins indicates the transcriptional network is frequently targeted for dis-regulation by both germline (BAP1, fumarate hydratase $(F H)$, neurofibromin 1 (NF1), succinate dehydrogenases (SDHA, $S D H B), V H L)^{46}$ and somatic mutations ( $\left.A R, I D H 2,{ }^{47} K D M 6 A, T E T 2\right)$. The mutations that converge on the TET2-AR nexus and TET2 co-expression network indicate tumors that share a common defect associated with 2-OG/succinate metabolism.

We defined a nexus of interacting cancer proteins with functions relevant to cancer, enriched in proteins encoded by $\mathrm{X}$ chromosome loci and single copy in males that indicates additional epigenetic modifications likely involved in androgen-AR signaling. Epigenetic modifications with varying half-lives would provide a mechanism to encode pathway signals for short and long term regulation of cellular processes. The nexus is enriched in enzymes that alter histones, DNA and RNA, indicating the histone code ${ }^{51,52}$ should incorporate nucleic acid modifications.

TET2 KD increases the proliferation of androgen-dependent $\mathrm{LNCaP}$ and androgenindependent DU145 cells, supporting that TET2 functions downstream in the androgen dependency pathway. Thus, therapy targeting TET2 loss may be effective in PCa with upstream AR alterations. Reduced TET2 expression is more prevalent in tumors that have progressed to metastatic disease and have a higher Gleason score, and is associated with significantly reduced DFS in PCa similar to MPDs. ${ }^{25-27,53}$ These results complement those of Takayama et al. ${ }^{37}$ who found that high expression of miR-29b targets TET2 and predicts poor outcome and decreased expression of miR-29b increased TET2 expression and reduced tumor growth in an animal model of PCa. Thus, reduced TET2 expression in prostate tumors may be an informative biomarker to identify patients likely to progress to metastatic disease.

\section{MATERIALS AND METHODS}

\section{Genotyping}

Genotyping utilized the 2.5M Bead Array (Illumina, San Diego, CA, USA). SNP genotyping in cell lines utilized primers as shown in Supplementary Table S3, standard PCR conditions, Big Dye sequencing reagents and a 3730 Genetic Analyzer (Life Technologies).

\section{Cell culture and transfection}

LNCaP cells were cultured in RPMI 1640 (Life Technologies) with 10\% fetal bovine serum. In experiments examining effects of DHT, charcoal stripped FBS (Life technologies) was substituted. Cells $\left(2 \times 10^{5}\right)$ were seeded into six-well plates and grown to $70-80 \%$ confluency before treatment with scRNA and siTET2-1 and transfection reagent (Santa Cruz Biotechnology, Dallas, TX, USA). Lipofectamine 3000 (Life technologies) was used for siTET2-4 transfections. Cell-based assays are in the Supplementary Materials and Methods. HEK293T cells were cultured to $60 \%$ confluency in Dulbecco's modified Eagle's medium with $10 \%$ fetal bovine serum (ClonTech, Mountain View, CA, USA) and $100 \mu \mathrm{g} / \mathrm{mL}$ penicillin/streptomycin. Cells were transfected with a construct expressing an N-terminal $3 x$-FLAG-TET2 using Fugene HD (Roche, Indianapolis, IN, USA) and were grown for an additional $72 \mathrm{~h}$ in a $5 \% \mathrm{CO}_{2} / 95 \%$ air-humidified incubator at $37{ }^{\circ} \mathrm{C}$. 


\title{
Affinity purification
}

3x-FLAG-TET2 was purified using a mouse anti-FLAG M2 monoclonal Ab covalently bound to agarose beads (Sigma-Aldrich, St. Louis, MO, USA). The eluate was analyzed by nanoflow reverse-phase liquid chromatography-tandem MS. Additional details are included in the Supplementary Materials and Methods.

\section{Gene expression}

TET2a and TET2a-delex2 expression were measured using FAM-labeled Hs00969056 and Hs01061044, respectively (Life Technologies). TET2b expression used TET2b-specific exon 3 primers and a FAM-labeled probe (Supplementary Table S3). Expression was normalized to HPRT1 (Hs02800695, Life Technologies) using the delta $\mathrm{C}_{\mathrm{T}}$ method to determine relative gene expression based on a human universal control of $100 \%$ (Clontech). cDNA from TET2 KD cells was prepared using a Cells-to-Ct kit (Ambion/Life Technologies, Foster City, CA, USA). Assays for individual genes (Applied Biosystems, Waltham, MA, USA) and the AR array (\#10047227, BioRad) were purchased. Expression was normalized by $\beta$-actin (Applied Biosystems) or the geometric mean of HPRT1 and GAPDH(AR array). See the Supplementary Materials and Methods for details about the co-expression analyses.

\section{Clinical impact of TET2 loss}

Samples included 29 NAT, 131 primary tumors and 19 metastatic tumors. ${ }^{9}$ Stage and grade were available for 14 and 18 metastatic tumors, respectively.

\section{Statistical analysis}

TET2 expression differences between clinical groups was assessed using a two-tailed Wilcoxon rank sum test, TET2 isoform and gene panel expression by a two-tailed T-test, and DFS by a log-rank test, all using R (www.R-project.org).

\section{Supplementary Material}

Refer to Web version on PubMed Central for supplementary material.

\section{Acknowledgments}

Financial Support: This work is supported in part by the Intramural Research Program of the National Institutes of Health, the National Cancer Institute and by Leidos Biomedical Research, Inc., under contract \# HHSN261200800001E.

\begin{abstract}
We thank Drs. Berton Zbar, Steve Bova and Tammy Naab for comments; and Lisa Garland, Mary Thompson, and Allen Kane for technical assistance. This work is supported in part by the Intramural Research Program of the National Institutes of Health, the National Cancer Institute, and by Leidos Biomedical Research, Inc., under contract \# HHSN261200800001E. The content of this publication does not necessarily reflect the views or policies of the Department of Health and Human Services, nor does mention of trade names, commercial products, or organizations imply endorsement by the U.S. government. The funders had no role in study design, data collection and analysis, decision to publish, or preparation of the manuscript.
\end{abstract}

\section{References}

1. Jemal A, Bray F, Center MM, Ferlay J, Ward E, Forman D. Global cancer statistics. CA Cancer J Clin. 2011; 61:69-90. [PubMed: 21296855] 
2. Delhommeau F, Dupont S, Della V, James C, Trannoy S, Masse A, et al. Mutation in TET2 in myeloid cancers. N Engl J Med. 2009; 360:2289-2301. [PubMed: 19474426]

3. Saint-Martin C, Leroy G, Delhommeau F, Panelatti G, Dupont S, James C, et al. Analysis of the teneleven translocation 2(TET2) gene in familial myeloproliferative neoplasms. Blood. 2009; 114:1628-1632. [PubMed: 19564637]

4. Seshagiri S, Stawiski EW, Durinck S, Modrusan Z, Storm EE, Conboy CB, et al. Recurrent Rspondin fusions in colon cancer. Nature. 2012; 488:660-664. [PubMed: 22895193]

5. Sato Y, Yoshizato T, Shiraishi Y, Maekawa S, Okuno Y, Kamura T, et al. Integrated molecular analysis of clear-cell renal cell carcinoma. Nat Genet. 2013; 45:860-867. [PubMed: 23797736]

6. Brennan CW, Verhaak RG, McKenna A, Campos B, Noushmehr H, Salama SR, et al. The somatic genomic landscape of glioblastoma. Cell. 2013; 155:462-477. [PubMed: 24120142]

7. Setiawan VW, Schumacher F, Prescott J, Haessler J, Malinowski J, Wentzensen N, et al. Crosscancer pleiotropic analysis of endometrial cancer. Carcinogenesis. 2014; 35:2068-2073. [PubMed: 24832084]

8. Song F, Amos CI, Lee JE, Lian CG, Fang S, Liu H, et al. Identification of a melanoma susceptibility locus and somatic mutation in TET2. Carcinogenesis. 2014; 35:2097-2101. [PubMed: 24980573]

9. Taylor BS, Schultz N, Hieronymus H, Gopalan A, Xiao Y, Carver BS, et al. Integrative genomic profiling of human prostate cancer. Cancer Cell. 2010; 18:11-22. [PubMed: 20579941]

10. Grasso CS, Wu YM, Robinson DR, Cao X, Dhanasekaran SM, Khan AP, et al. The mutational landscape of lethal castration-resistant prostate cancer. Nature. 2012; 487:239-243. [PubMed: 22722839]

11. Nickerson ML, Im KM, Misner KJ, Tan W, Lou H, Gold B, et al. Somatic alterations contributing to metastasis of a castration-resistant prostate cancer. Hum Mutat. 2013; 34:1231-1241. [PubMed: 23636849]

12. Eeles RA, Kote-Jarai Z, Al Olama AA, Giles GG, Guy M, Severi G, et al. Identification of seven new prostate cancer susceptibility loci through a genome-wide association study. Nat Genet. 2009; 41:1116-1121. [PubMed: 19767753]

13. Pomerantz MM, Werner L, Xie W, Regan MM, Lee GS, Sun T, et al. Association of prostate cancer risk loci with disease aggressiveness and prostate cancer-specific mortality. Cancer Prev Res. 2011; 4:719-728.

14. Tonin P, Weber B, Offit K, Couch F, Rebbeck TR, Neuhausen S, et al. Frequency of recurrent BRCA1 and BRCA2 mutations in Ashkenazi Jewish breast cancer families. Nat Med. 1996; 2:1179-1183. [PubMed: 8898735]

15. Tomlins SA, Rhodes DR, Perner S, Dhanasekaran SM, Mehra R, Sun XW, et al. Recurrent fusion of TMPRSS 2 and ETS transcription factor genes in prostate cancer. Science. 2005; 310:644-648. [PubMed: 16254181]

16. Varela I, Tarpey P, Raine K, Huang D, Ong C-K, Stephens P, et al. Exome sequencing identifies frequent mutation of the SWI/SNF complex gene PBRM1 in renal carcinoma. Nature. 2011; 469:539-542. [PubMed: 21248752]

17. Pena-Llopis S, Christie A, Xie XJ, Brugarolas J. Cooperation and antagonism among cancer genes. Cancer Res. 2013; 73:4173-4179. [PubMed: 23832661]

18. Liu W, Laitinen S, Khan S, Vihinen M, Kowalski J, Yu G, et al. Copy number analysis indicates monoclonal origin of lethal metastatic prostate cancer. Nat Med. 2009; 15:559-565. [PubMed: 19363497]

19. Segil N, Roberts SB, Heintz N. Mitotic phosphorylation of the Oct-1 homeodomain and regulation of Oct-1 DNA binding activity. Science. 1991; 254:1814-1816. [PubMed: 1684878]

20. Obinata D, Takayama K, Urano T, Murata T, Kumagai J, Fujimura T, et al. Oct1 regulates cell growth of LNCaP cells and is a prognostic factor for prostate cancer. Int J Cancer. 2012; 130:1021-1028. [PubMed: 21387309]

21. Barbieri CE, Baca SC, Lawrence MS, Demichelis F, Blattner M, Theurillat JP, et al. Exome sequencing identifies recurrent $S P O P, F O X A 1$ and MED12 mutations in prostate cancer. Nat Genet. 2012; 44:685-689. [PubMed: 22610119]

22. Baca SC, Prandi D, Lawrence MS, Mosquera JM, Romanel A, Drier Y, et al. Punctuated evolution of prostate cancer genomes. Cell. 2013; 153:666-677. [PubMed: 23622249] 
23. Gao J, Aksoy BA, Dogrusoz U, Dresdner G, Gross B, Sumer SO, et al. Integrative analysis of complex cancer genomics and clinical profiles using the cBioPortal. Sci Signal. 2013; 6:pl1. [PubMed: 23550210]

24. Beroukhim R, Mermel CH, Porter D, Wei G, Raychaudhuri S, Donovan J, et al. The landscape of somatic copy-number alteration across human cancers. Nature. 2010; 463:899-905. [PubMed: 20164920]

25. Scopim-Ribeiro R, Machado-Neto JA, de Melo Campos P, Silva CA, Favaro P, Lorand-Metze I, et al. Ten-Eleven-Translocation 2 (TET2) is downregulated in myelodysplastic syndromes. Eur J Haematol. 2014; 94:413-418. [PubMed: 25200248]

26. Yang H, Liu Y, Bai F, Zhang JY, Ma SH, Liu J, et al. Tumor development is associated with decrease of TET gene expression and 5-methylcytosine hydroxylation. Oncogene. 2013; 32:663669. [PubMed: 22391558]

27. Ko M, Huang Y, Jankowska AM, Pape UJ, Tahiliani M, Bandukwala HS, et al. Impaired hydroxylation of 5-methylcytosine in myeloid cancers with mutant TET2. Nature. 2010; 468:839843. [PubMed: 21057493]

28. Chen Q, Chen Y, Bian C, Fujiki R, Yu X. TET2 promotes histone O-GlcNAcylation during gene transcription. Nature. 2013; 493:561-564. [PubMed: 23222540]

29. Lynch TP, Ferrer CM, Jackson SR, Shahriari KS, Vosseller K, Reginato MJ. Critical role of Olinked beta-N-acetylglucosamine transferase in prostate cancer invasion, angiogenesis, and metastasis. J Biol Chem. 2012; 287:11070-11081. [PubMed: 22275356]

30. Passon DM, Lee M, Rackham O, Stanley WA, Sadowska A, Filipovska A, et al. Structure of the heterodimer of human NONO and paraspeckle protein component 1 and analysis of its role in subnuclear body formation. Proc Natl Acad Sci USA. 2012; 109:4846-4850. [PubMed: 22416126]

31. Loy CJ, Sim KS, Yong EL. Filamin-A fragment localizes to the nucleus to regulate androgen receptor and coactivator functions. Proc Natl Acad Sci USA. 2003; 100:4562-4567. [PubMed: 12682292]

32. van Haaften G, Dalgliesh GL, Davies H, Chen L, Bignell G, Greenman C, et al. Somatic mutations of the histone H3K27 demethylase gene $U T X$ in human cancer. Nat Genet. 2009; 41:521-523. [PubMed: 19330029]

33. Guo G, Sun X, Chen C, Wu S, Huang P, Li Z, et al. Whole-genome and whole-exome sequencing of bladder cancer identifies frequent alterations in genes involved in sister chromatid cohesion and segregation. Nat Genet. 2013; 45:1459-1463. [PubMed: 24121792]

34. Dong X, Sweet J, Challis JR, Brown T, Lye SJ. Transcriptional activity of androgen receptor is modulated by two RNA splicing factors, PSF and p54nrb. Mol Cell Biol. 2007; 27:4863-4875. [PubMed: 17452459]

35. Norris JD, Chang CY, Wittmann BM, Kunder RS, Cui H, Fan D, et al. The homeodomain protein HOXB13 regulates the cellular response to androgens. Mol Cell. 2009; 36:405-416. [PubMed: 19917249]

36. Lilja H, Ulmert D, Vickers AJ. Prostate-specific antigen and prostate cancer: prediction, detection and monitoring. Nat Rev Cancer. 2008; 8:268-278. [PubMed: 18337732]

37. Takayama K, Misawa A, Suzuki T, Takagi K, Hayashizaki Y, Fujimura T, et al. TET2 repression by androgen hormone regulates global hydroxymethylation status and prostate cancer progression. Nat Commun. 2015; 6:8219. [PubMed: 26404510]

38. Robinson D, Van Allen EM, Wu YM, Schultz N, Lonigro RJ, Mosquera JM, et al. Integrative clinical genomics of advanced prostate cancer. Cell. 2015; 161:1215-1228. [PubMed: 26000489]

39. Cancer Genome Atlas Research Network. The Molecular Taxonomy of Primary Prostate Cancer. Cell. 2015; 163:1011-1025. [PubMed: 26544944]

40. Pena-Llopis S, Vega-Rubin-de-Celis S, Liao A, Leng N, Pavia-Jimenez A, Wang S, et al. BAP1 loss defines a new class of renal cell carcinoma. Nat Genet. 2012; 44:751-759. [PubMed: 22683710]

41. Nickerson ML, Dancik GM, Im KM, Edwards MG, Turan S, Brown J, et al. Concurrent alterations in TERT, KDM6A, and the BRCA pathway in bladder cancer. Clin Cancer Res. 2014; 20:49354948. [PubMed: 25225064] 
42. Zhang B, Horvath S. A general framework for weighted gene co-expression network analysis. Stat Appl Genet Mol Biol. 2005; 4 Article17, Epub 2005 Aug 12.

43. Sharan R, Ulitsky I, Shamir R. Network-based prediction of protein function. Mol Syst Biol. 2007; 3:88. [PubMed: 17353930]

44. Hu L, Li Z, Cheng J, Rao Q, Gong W, Liu M, et al. Crystal structure of TET2-DNA complex: insight into TET-mediated 5mC oxidation. Cell. 2013; 155:1545-1555. [PubMed: 24315485]

45. Hu L, Lu J, Cheng J, Rao Q, Li Z, Hou H, et al. Structural insight into substrate preference for TET-mediated oxidation. Nature. 2015; 527:118-122. [PubMed: 26524525]

46. Linehan MW, Srinivasan R, Schmidt LS. The genetic basis of kidney cancer: a metabolic disease. Nat Rev Urol. 2010; 7:277-285. [PubMed: 20448661]

47. Figueroa ME, Abdel-Wahab O, Lu C, Ward PS, Patel J, Shih A, et al. Leukemic IDH1 and IDH2 mutations result in a hypermethylation phenotype, disrupt TET2 function, and impair hematopoietic differentiation. Cancer Cell. 2010; 18:553-567. [PubMed: 21130701]

48. Sérandour AA, Avner S, Oger F, Bizot M, Percevault F, Lucchetti-Miganeh C, et al. Dynamic hydroxymethylation of deoxyribonucleic acid marks differentiation-associated enhancers. Nucleic Acids Res. 2012; 40:8255-8265. [PubMed: 22730288]

49. Hsieh CL, Fei T, Chen Y, Li T, Gao Y, Wang X, et al. Enhancer RNAs participate in androgen receptor-driven looping that selectively enhances gene activation. Proc Natl Acad Sci USA. 2014; 111:7319-7324. [PubMed: 24778216]

50. Fu L, Guerrero CR, Zhong N, Amato NJ, Liu Y, Liu S, et al. Tet-mediated formation of 5hydroxymethylcytosine in RNA. J Am Chem Soc. 2014; 136:11582-11585. [PubMed: 25073028]

51. Strahl B, Allis C. The language of covalent histone modifications. Nature. 2000; 403:41-45. [PubMed: 10638745]

52. Jenuwein T, Allis C. Translating the histone code. Science. 2001; 293:1074-1080. [PubMed: 11498575]

53. Metzeler KH, Maharry K, Radmacher MD, Mrózek K, Margeson D, Becker H, et al. TET2 mutations improve the new European LeukemiaNet risk classification of acute myeloid leukemia: a Cancer and Leukemia Group B study. J Clin Oncol. 2011; 29:1373-1381. [PubMed: 21343549] 
a

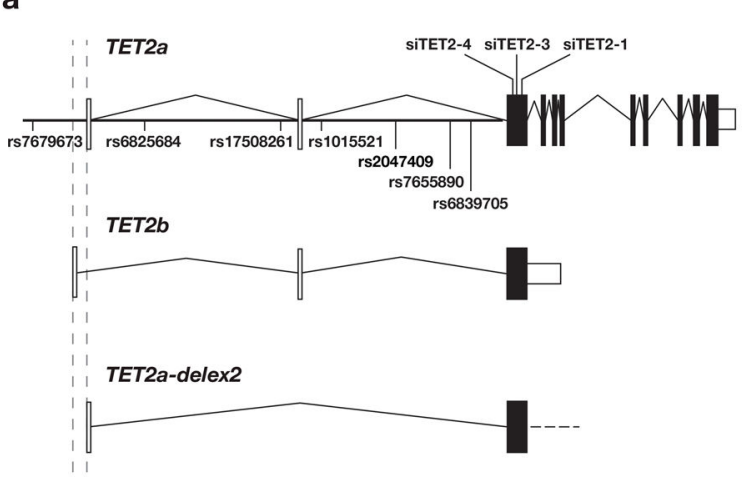

b

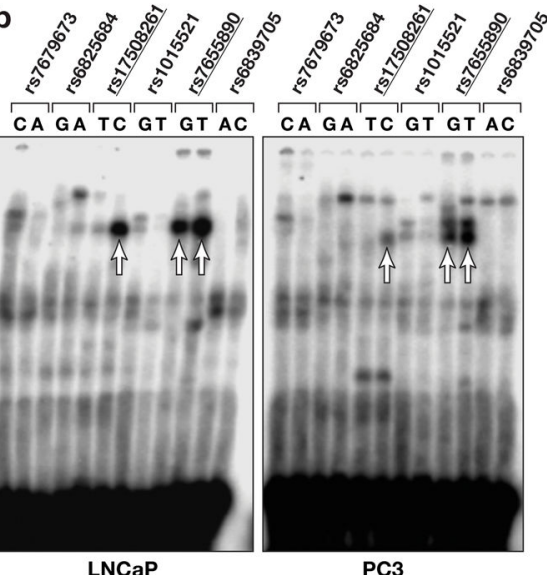

C
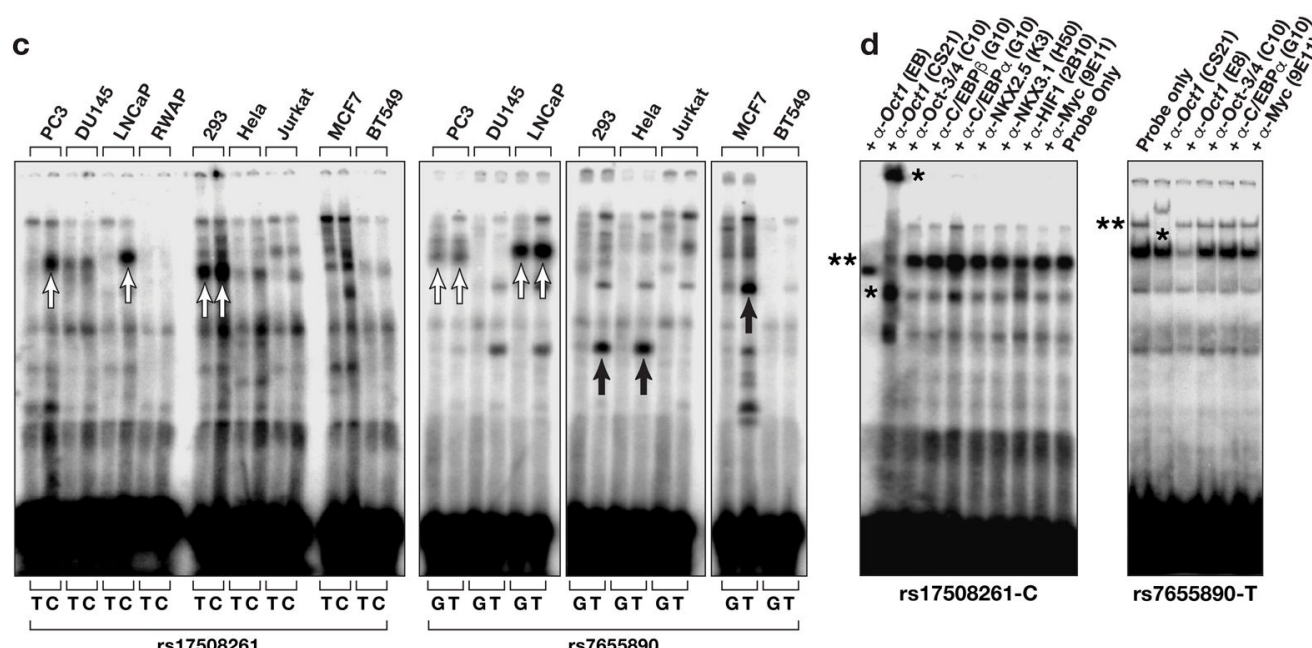

rs17508261-C

rs7655890-T

e
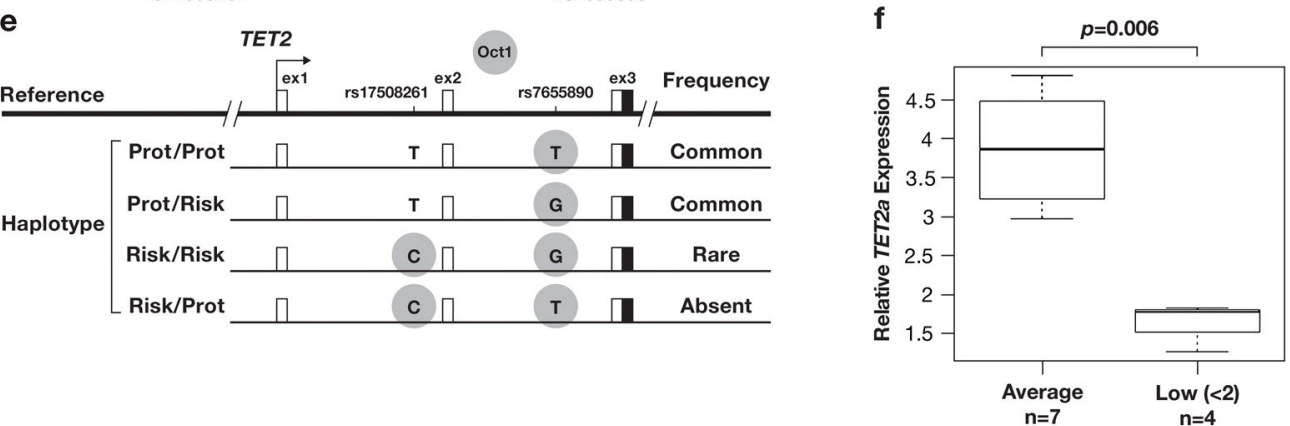

Figure 1.

PCa risk SNPs. (a) Risk SNPs and TET2 isoforms. Locations of risk SNPs and binding sites of siRNAs used in this study are indicated. Vertical dotted line, alternative first exons; horizontal dotted line (TET2a-delex2), transcript structure not determined; white, noncoding; black, protein-coding. (b) Electrophoretic mobility shift assays show nuclear protein binding to rs17508261-C and rs7655890-G/T oligonucleotides in PCa cell line nuclear extracts (arrow). (c) Nuclear proteins associate with oligonucleotides containing the indicated variant in repeat experiments and extracts from additional cell lines (arrows). Black arrows, not further examined. (d) Supershift assays in the presence of TF antibodies 
show altered complex migration in the presence of anti-Oct1 $(*)$ compared with probe alone (**). (e) A rare risk SNP haplotype (risk/risk) binds Oct1 (grey circle). Prot, protective. (f) TET2a expression is reduced in a subset of prostate cell lines (3.87 \pm 0.75 [average] versus $1.67 \pm 0.27$ [low]). Expression analyzed in triplicate; p-value, two-tailed Wilcoxon rank sum test; error bars, mean \pm SD. 

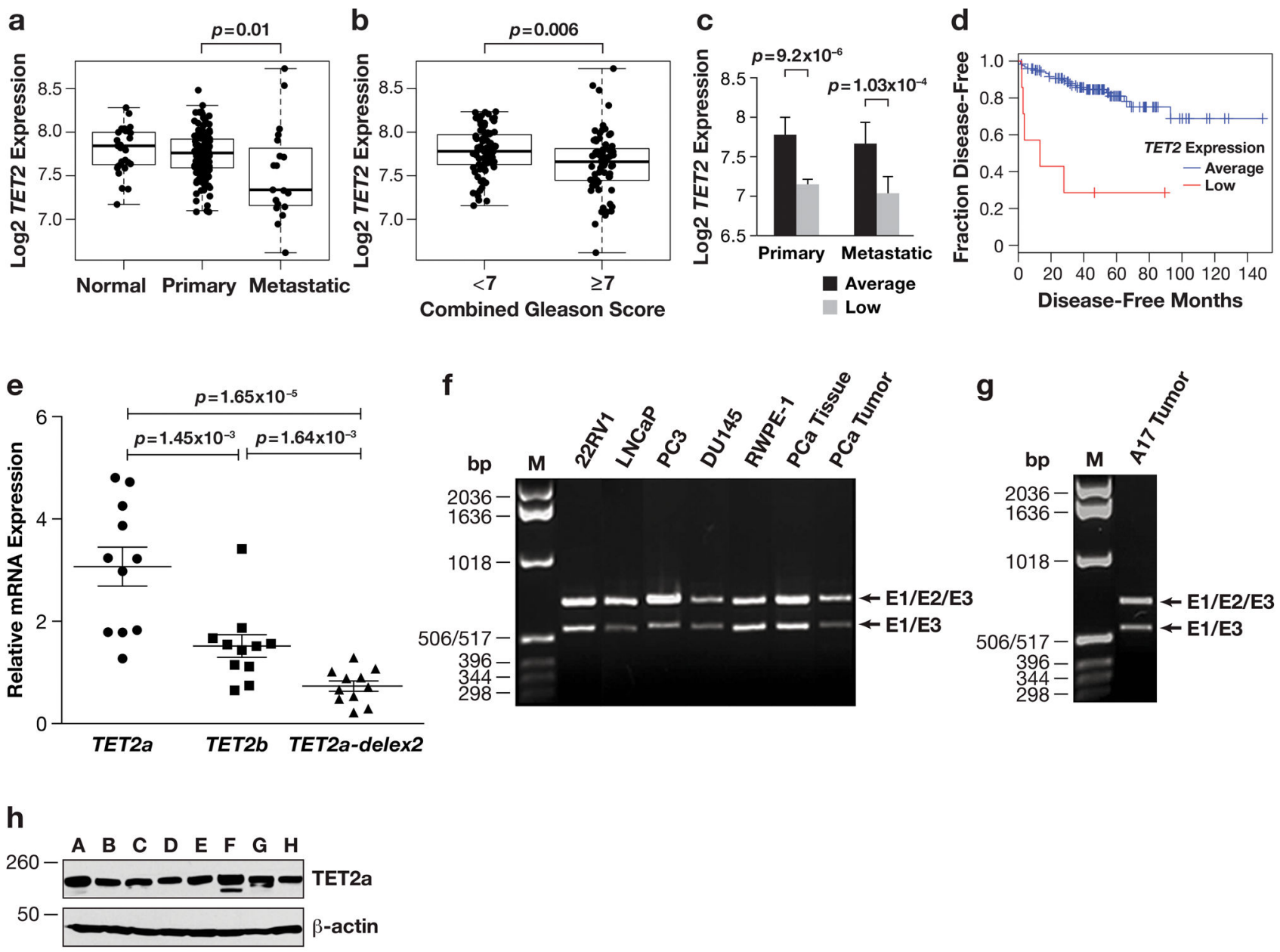

Figure 2.

TET2 alterations and expression in prostate cancer. Reduced TET2 expression in subsets of (a) metastatic tumors; (b) high ( $\geq 7$ ) Gleason score tumors; (c) primary and metastatic tumors; two-tailed Wilcoxon rank sum test; and (d) tumors from patients with reduced DFS ( $\mathrm{p}=6.4 \times 10^{-6} ; \log$ rank test). (e) TET2a is the most highly expressed transcript in normal prostate tissue ( $\mathrm{n}=11$, two-tailed paired T-test) as shown by QPCR performed in triplicate. (f) TET2a containing exons 1-3 (E1/E2/E3) and TET2a-delex 2 containing exons 1 and 3 (E1/E3) are expressed in all prostate samples as shown by RT-PCR. (g) TET2a and TET2adelex 2 are expressed in RNA from a PCa patient with a somatic TET2 mutation. (h) TET2 protein in cell lines as shown by WB using TET2 antibody, MAb-179-050 (Diagenode, Denville, NJ, USA); PCa unless indicated: A, VCaP; B, 22RV1; C, HeLa (cervix); D, LNCaP; E, PC3; F, DU145; G, MCF7 (breast); H, HEK293T (kidney). Note the additional band in the DU145 lysate, a cell line with a TET2 p.T229fs* mutation. Left, molecular weight $(\mathrm{MW})$ in kiloDaltons $(\mathrm{kD})$. Error bars, mean $\pm \mathrm{SD}$. 

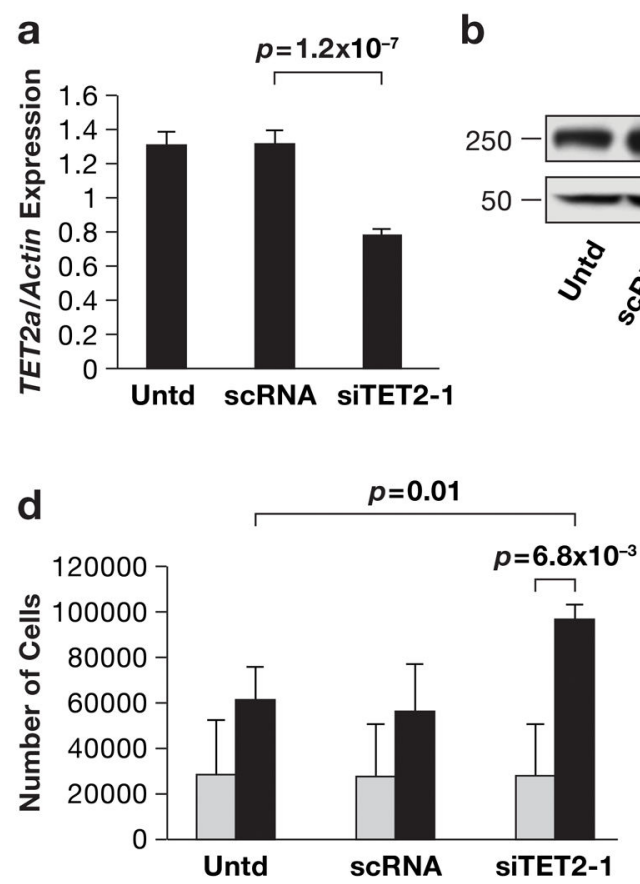

f

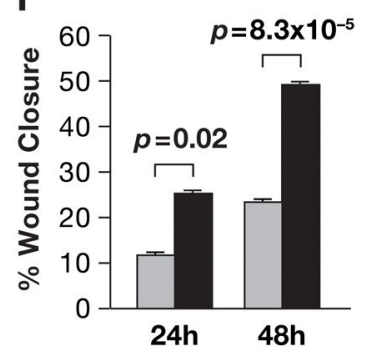

g

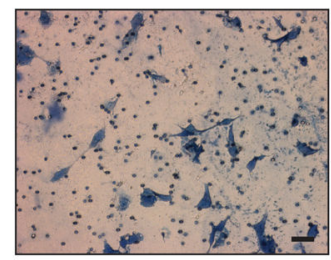

b

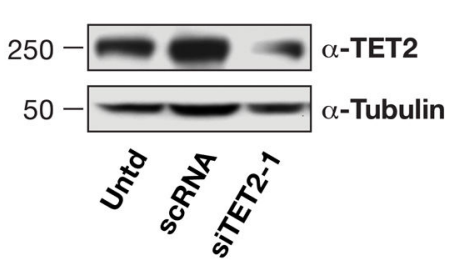

C

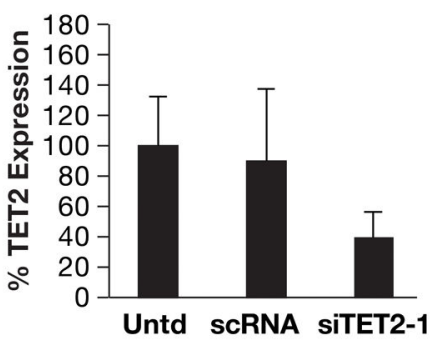

e
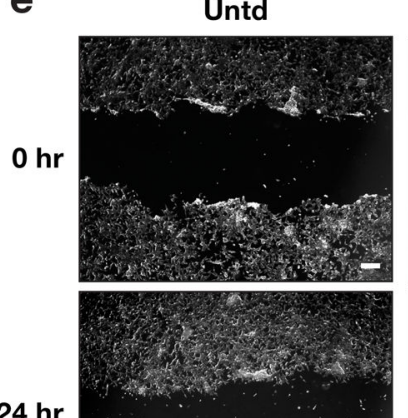

$24 \mathrm{hr}$
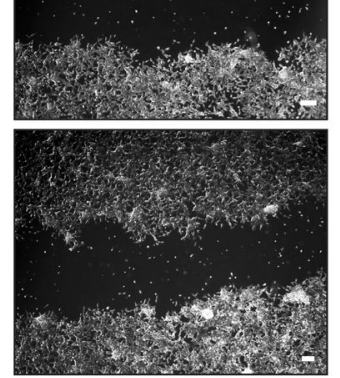

SITET2-1
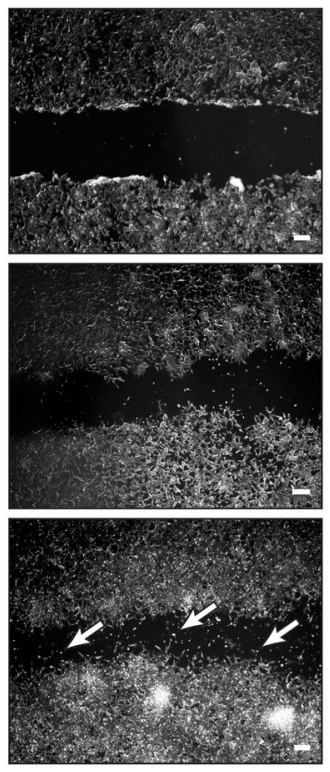

h

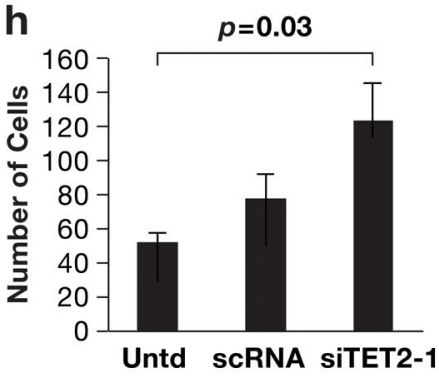

Figure 3.

TET2 loss is associated with a cancer phenotype. (a) Reduced TET2a at $24 \mathrm{~h}$ after siTET2-1 treatment across six biological replicates; p-value, one-tailed T-test. (b) Reduced TET2 (aTET2) at $24 \mathrm{~h}$ by WB. (c) Quantitated WBs in triplicate at $24 \mathrm{~h}$. Controls not shown. (d) TET2 KD (siTET2) increases in vitro LNCaP cell proliferation at $24 \mathrm{~h}$ (black) from time 0 (gray); p-value, two-tailed T-test. (e,f) wound healing at 24 and 48h; p-value, two-tailed Ttest. (g,h) transwell invasion; p-value, two-tailed T-test. Gross cell morphology appears unchanged after the invasion assay. Untd, untreated; scRNA, scrambled siRNA treatment; 
siTET2, siTET2-1 treatment; size bar, $100 \mu \mathrm{m}$; error bars, mean \pm SD. All experiments were performed in triplicate using $40 \mathrm{nM}$ siTET2-1. TET2 antibody: MAb-179-050, Diagenode, Denville, NJ, USA. 

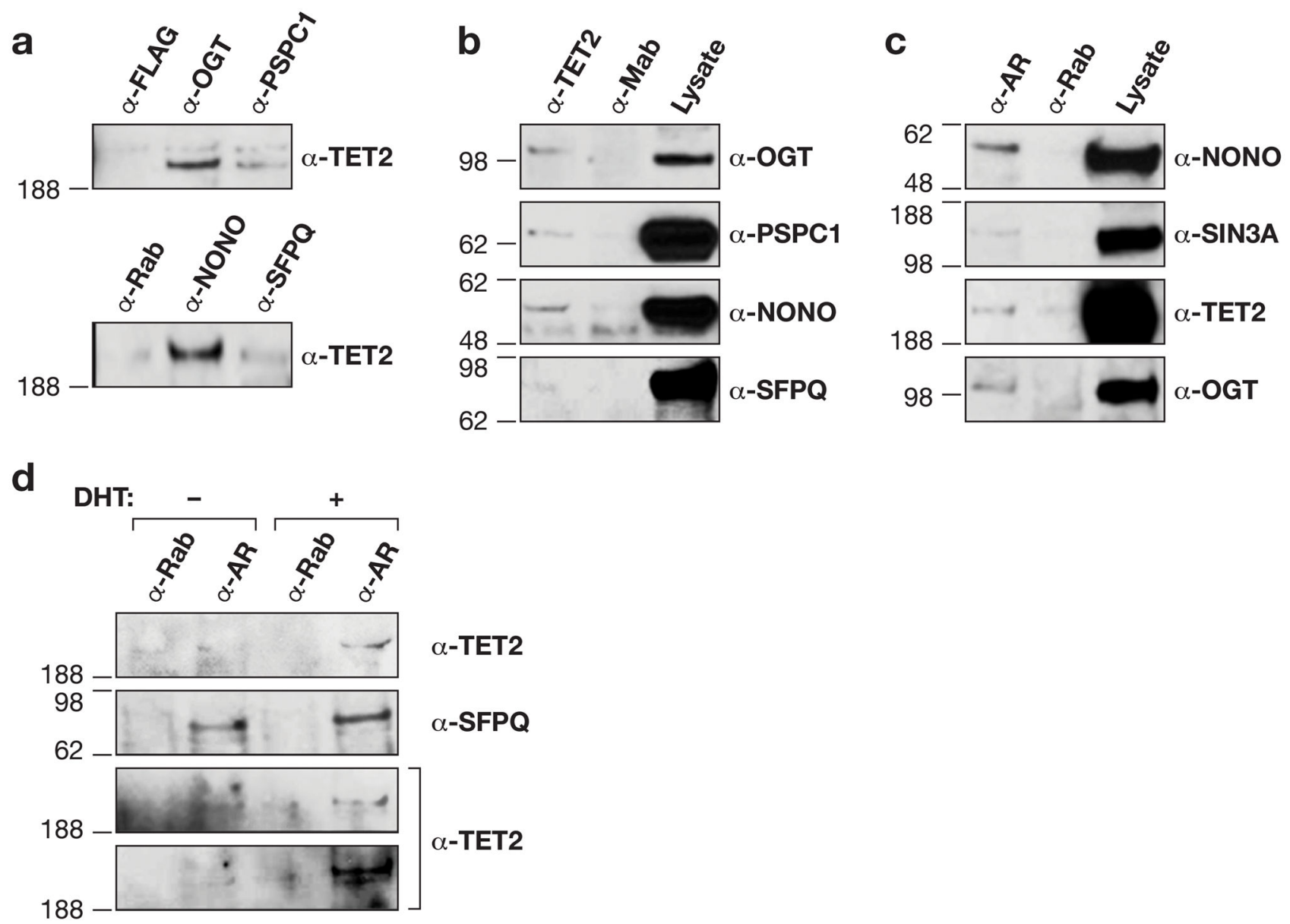

Figure 4.

Endogenous TET2 interactors in LNCaP cells. (a) Top, IP with a-OGT or a-PSPC1, but not a-FLAG, precipitates endogenous TET2 (a-TET2). Bottom, IP with a-NONO or a-SFPQ, not a-Rab, precipitates endogenous TET2. (b) IP with a-TET2, not anti-mouse IgG (a$\mathrm{Mab}$ ), precipitates endogenous OGT (a-OGT), PSPC1 (a-PSPC1), NONO (a-NONO) and SFPQ (a-SFPQ). (c) IP with a-AR, not a-Rab, precipitates endogenous NONO, SIN3A ( $a-$ SIN3A), TET2 and OGT. (d) DHT (+DHT) and IP with a-AR, not a-Rab, precipitates endogenous TET2 and SFPQ. Experiment 1 (top), row 1 and 2, short exposure; experiment 2, row 3, short exposure; row 4, long exposure. Left, molecular weight in $\mathrm{kD}$. Lysate, total protein. 

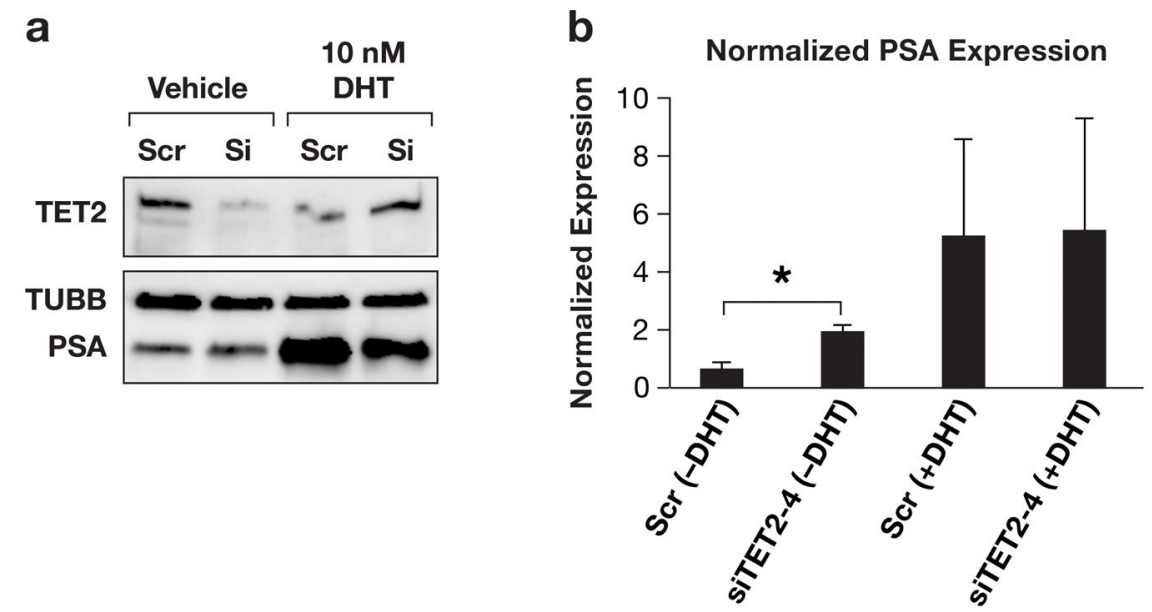

C

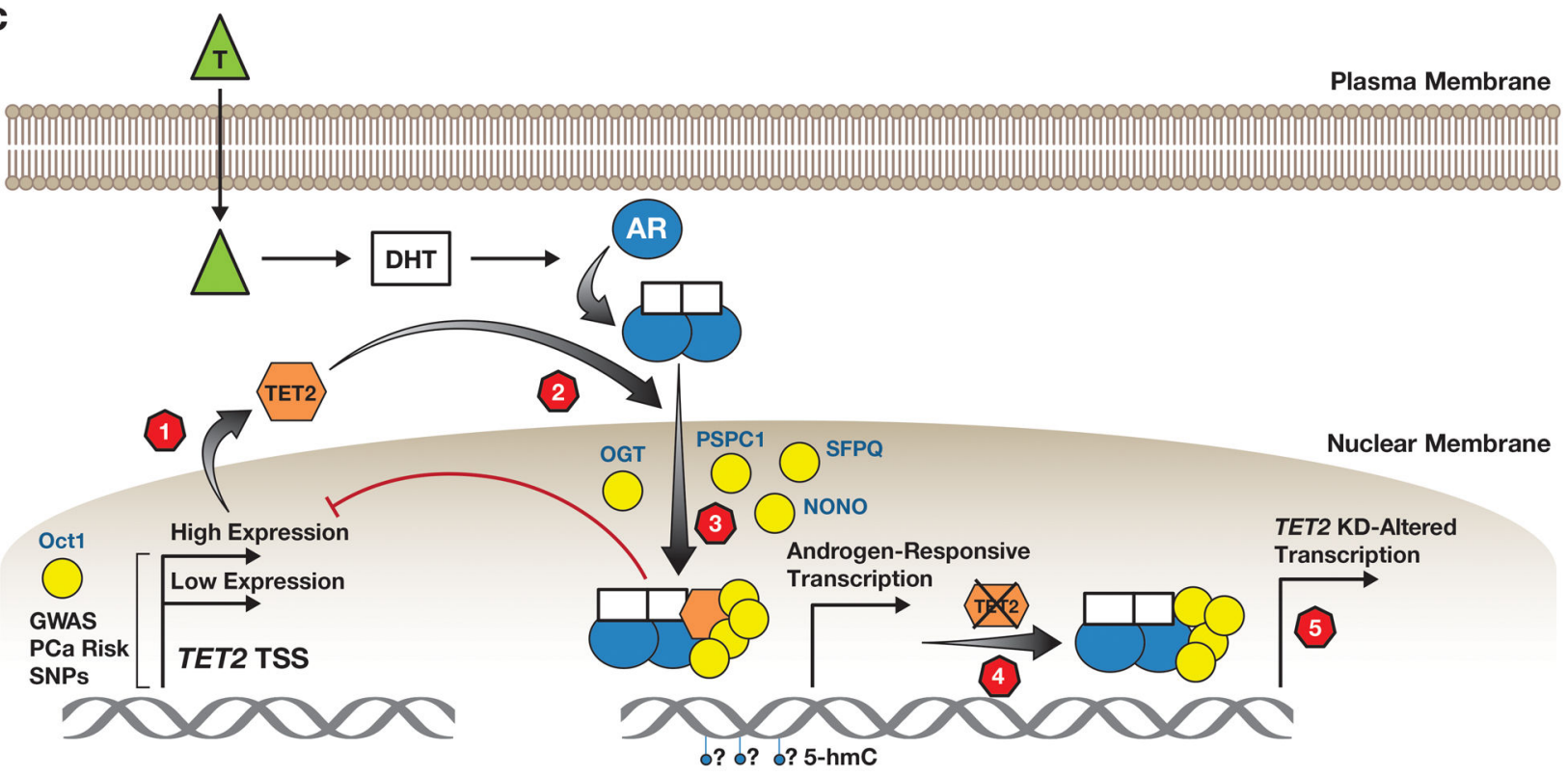

Figure 5. PSA increases in response to TET2 KD and DHT treatment

(a) LNCaP cells were treated with 40nM siTET2-4 directed against TET2 ( $\mathrm{Si}$ ) or low GC content scrambled siRNA (Scr) as a control. DHT (or ETOH vehicle) was added to $10 \mathrm{nM}$ final concentration $48 \mathrm{hr}$ post siRNA transfection; $24 \mathrm{hr}$ post-hormone treatment, cells were lysed and processed for Western blot analysis. A representative of 3 Western blots is shown, which were analyzed by densitometry with ImageJ software. (b) Loss of TET2 or treatment with DHT increases PSA protein levels. TUBB, b-tubulin; T, testosterone; DHT, dihydrotestosterone; *, $\mathrm{p}=5 \times 10^{-3}$, two-tailed T-test; error bars, mean $\pm \mathrm{SD}$. (c) A hypothetical model of TET2-AR signaling: (1) Germline PCa risk SNPs influence TET2 expression. (2) TET2 binds the AR. (3) A TET2-AR complex may mediate gene expression changes potentially via DNA 5-hmC (lollipop). (4) TET2 loss depletes TET2. (5) Altered transcription facilitates PCa progression. T, testosterone; DHT, dihydrotestosterone. 
a

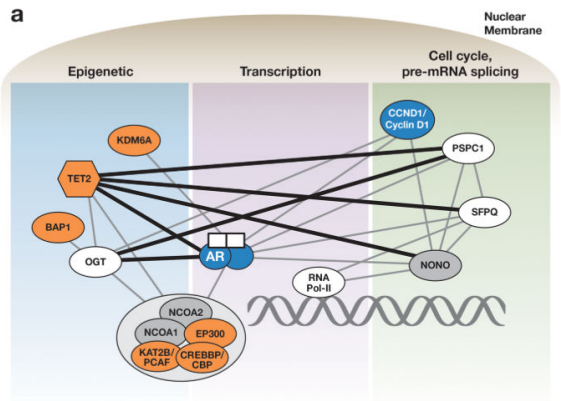

b
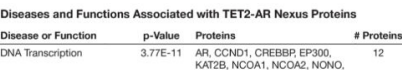

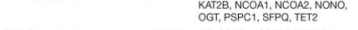

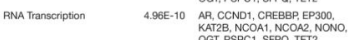

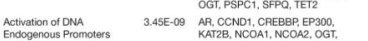

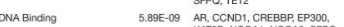

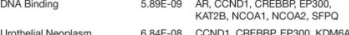

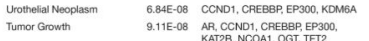

Sexual Betavior 9.795 -08 AR, CREBBP? NCOA1, NCOA2

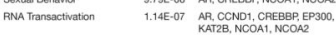

Rubinsten-Taybi Sydrome 3.77E-07 CAEBBP EP300 -

Desquanation of Germ Colls 3.77E-07 NCOA1, NCOA3 Onset of Degeneration of $3.77 \mathrm{E}-07$ NCOA1, NCON

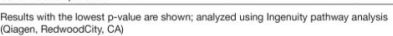

c

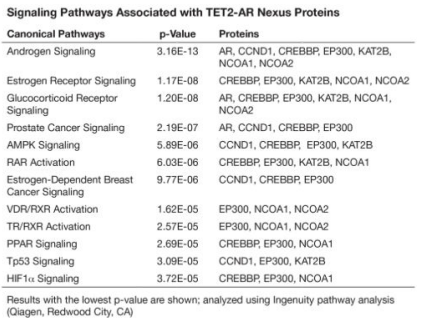

d

KMT2C/
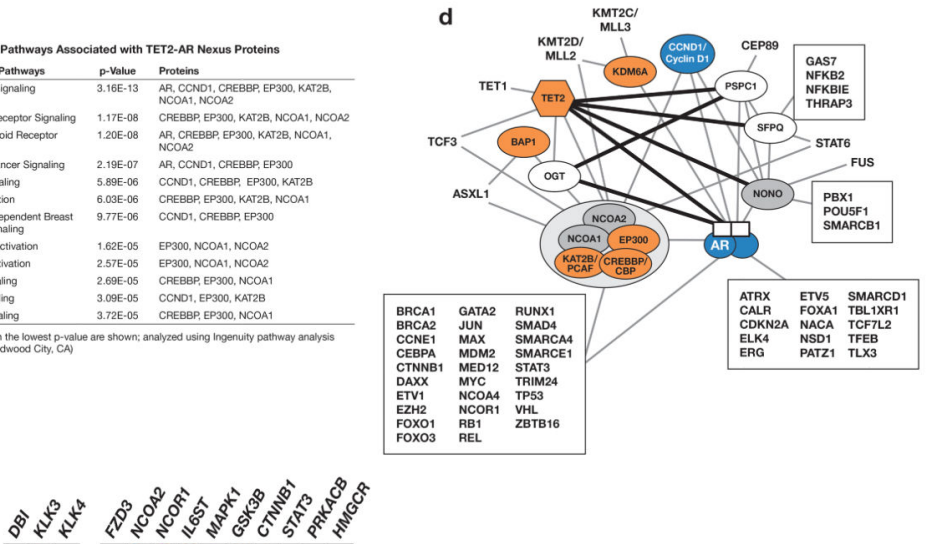

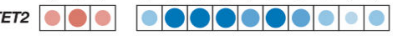

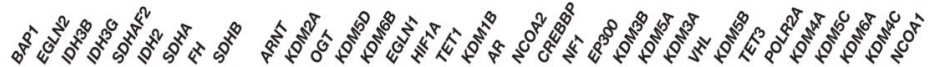

TEт2

Figure 6.

TET2-AR is associated with proteins encoded by frequently altered cancer genes. (a) Protein functions. (b) Associated disease functions. (c) Associated signaling pathways. (d) Interacting cancer proteins from the Cancer Gene Census (COSMIC; August 2015). Black line, interactions, this study; blue, oncogene; orange, tumor suppressor; grey, uncertain; white, not frequently altered in cancer. Co-expressed androgen-AR genes (e) and 2-OG/ succinate-associated genes (f) whose expression is anti-correlated (red dots) or correlated (blue dots) with TET2 by Pearson correlation in TCGA PAD tumors based on RNAsequencing available through the cBioPortal (Broad Institute, Cambridge, MA). A genegene expression correlation is indicated by a circle if the Pearson correlation p-value $<0.01$; the colored scoring index is included in Supplementary Figure S10; the gene order is based on a hierarchical clustering method. 


\section{롤 \\ }

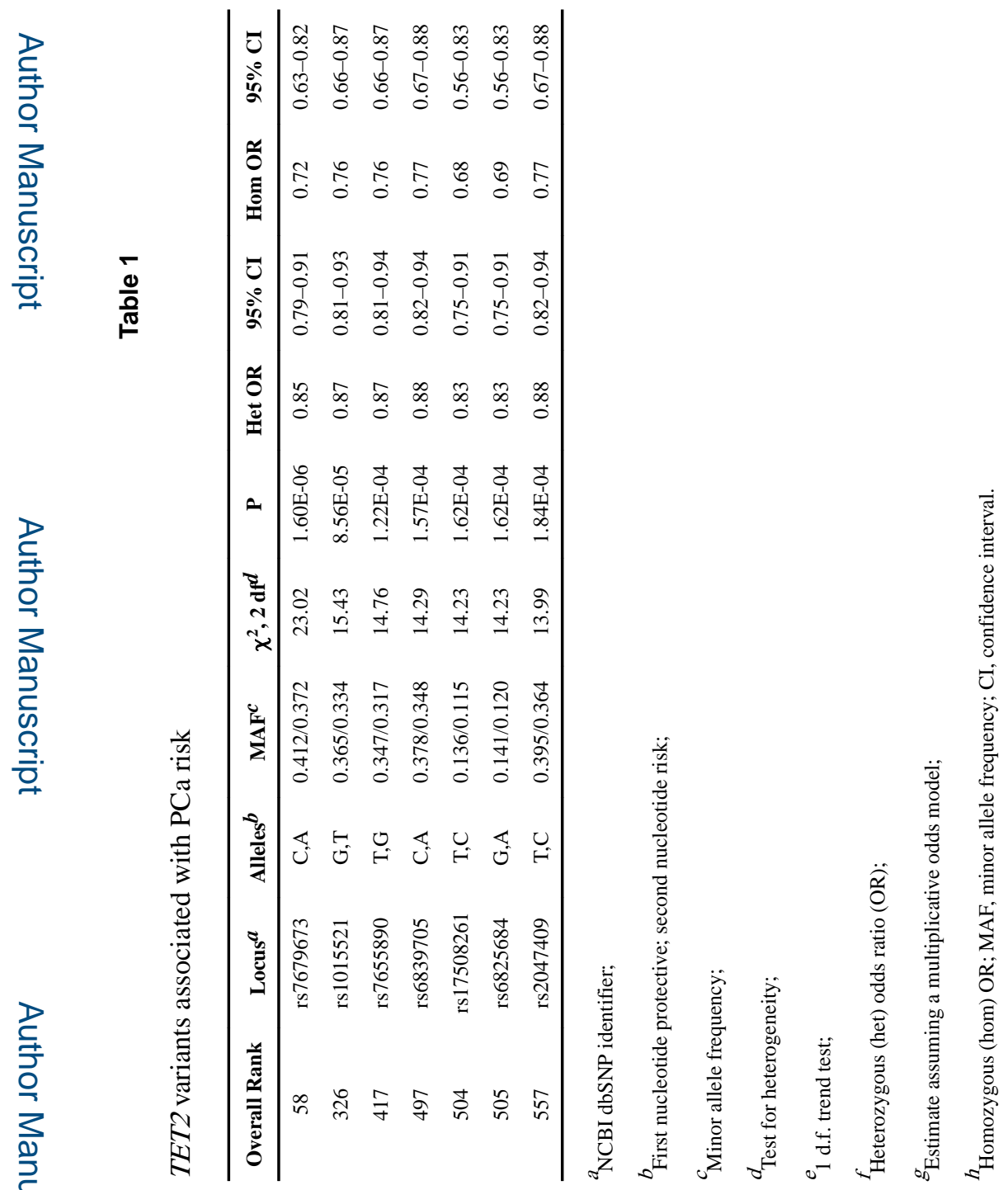




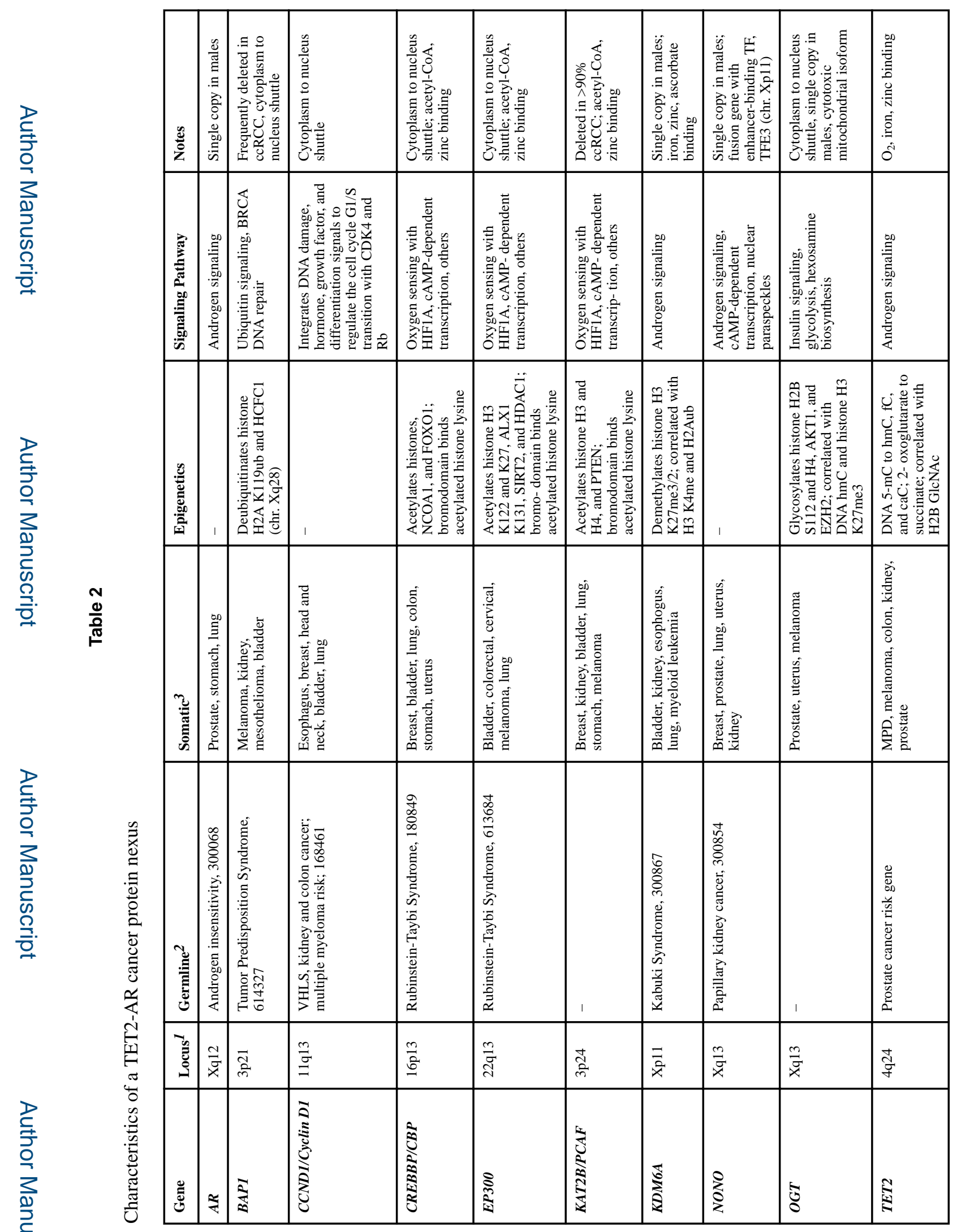




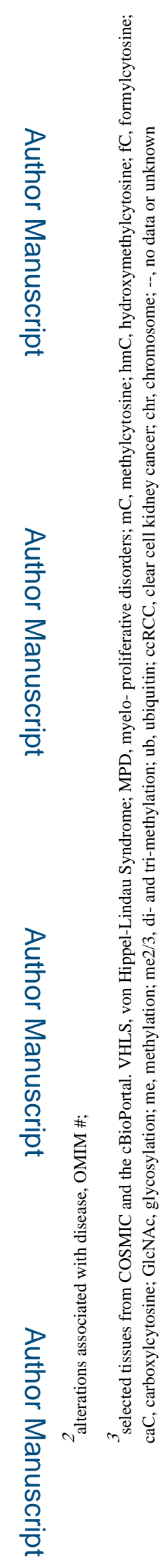

Oncogene. Author manuscript; available in PMC 2017 May 07. 